\title{
Characterization of a plankton community in a fish farm
}

\author{
Caracterização da comunidade planctônica em sistema de piscicultura
}

Lúcia Helena Sipaúba Tavares ${ }^{1}$, Rodrigo Ney Millan ${ }^{1}$ and Rachel Magalhães Santeiro ${ }^{2}$

${ }^{1}$ Laboratório de Limnologia e Produção de Plâncton, Centro de Aquicultura, Universidade Estadual Paulista - UNESP, CEP 14884-900, Jaboticabal, SP, Brazil e-mail: sipauba@caunesp.unesp.br, rodrigomillan@yahoo.com.br

${ }^{2}$ Instituto Superior de Ciências e da Saúde - INCISA, Av. Barão Homem de Melo 4324, 1 andar, CEP 30450-250, Belo Horizonte, MG, Brazil e-mail: rachelsanteiro@yahoo.com.br

\begin{abstract}
Aim: The objective of this study was to analyze the composition of phytoplankton and zooplankton communities related to the dynamics of a fish farm; Methods: Samples were collected every other day, within a period of twenty consecutive days, during the dry and rainy seasons. Two samples were collected upstream from the fish pond (reservoir); the other four samples were collected in the fish farm area; Results: Rotifera and Chlorophyceae species were found in high densities at almost all sampling sites during both seasons under analysis. The higher phytoplankton species richness from site $\mathrm{P}_{3}$ to $\mathrm{P}_{6}$ was influenced by the management employed within the fish farm. The zooplankton community showed low values of density, species richness and equitability during the dry season; Conclusions: The continuous water flow and the addition of fertilizers (organic and inorganic) in the fish ponds had an influence on the plankton community, leading to a reduction in water quality and Cyanobacteria dominance.
\end{abstract}

Keywords: fish ponds, plankton, aquaculture, diversity, richness.

Resumo: Objetivo: O presente estudo objetivou conhecer a composição das comunidades fitoplanctônica e zooplanctônica em função da dinâmica de uma estação de piscicultura; Métodos: Foram realizadas amostragens a cada dois dias, por vinte dias consecutivos em duas épocas do ano: seca e chuvosa, em dois pontos a montante da piscicultura (reservatório) e outros quatro dentro da piscicultura; Resultados: As espécies de Rotifera e de Chlorophyceae foram encontradas em maior densidade nos dois períodos estudados, em quase todos os pontos de coleta. Elevada riqueza de espécies fitoplanctônicas foi observadas a partir do $\mathrm{P}_{3}$ até $\mathrm{P}_{6}$, influenciada pelo manejo empregado dentro da estação de piscicultura. A comunidade zooplanctônica mostrou baixos valores de densidade, riqueza de espécies e equitabilidade durante a estação seca; Conclusóes: $\mathrm{O}$ fluxo contínuo de água e a adição de fertilizantes (orgânico e inorgânico) nos tanques de piscicultura influenciaram a comunidade planctônica com empobrecimento da qualidade da água e dominância de Cyanobacteria.

Palavras-chave: viveiros, plâncton, aquicultura, diversidade, riqueza.

\section{Introduction}

The plankton community is one of the main sources of energy flow in water environments. Its composition and density are relevant to detect-albeit partially-changes in the environment. Fluctuations in plankton communities in fish farms indicate the organisms' dependence on the physical and chemical conditions and on the management employed, which lead to great oscillations caused by the very dynamics of the fish ponds. Human activities are a highly determining factor in the limnology of these artificial shallow systems and in establishing their productivity.
The overall objective of organic and inorganic fertilizers is to increase fish farm productivity. A common practice in fish farms is the fertilization of ponds to enhance algae growth and promote the production of zooplankton suitable for larvae.

Several studies carried out in freshwater environments have established that the growth of phytoplankton may be controlled to a large degree by the limitation of nutrients, availability of light and the composition and abundance of zooplankton (Basualto et al., 2006). 
The structure and functioning of the zooplankton community with regard to species composition and the processes that control changes in the density of each species are affected by several factors, such as the nature and availability of food resources, types of predatory interaction in the water environment, physical and chemical aspects of water, and anthropogenic changes.

In tropical shallow water systems, the role of rainy and dry seasons, and mixing have a greater impact on plankton biomass than inter-seasonal variations (Zimba et al., 2003).

Due to management in fish culture, a high level of the farm's primary productivity is maintained. As a rule, productivity in meso-oligotrophic farms is positively correlated with the richness of zooplankton species, whereas primary productivity is correlated with changes in the composition of zooplankton species (Dodson et al., 2009).

Analysis of the plankton community in fish farm systems is an important tool to evaluate water quality conditions, as changes in nutrient concentrations determine changes in species composition. The current study analyzes the influence of management adopted at the fish farm in the structure (composition and density) phytoplankton and zooplankton communities at six different sites that receive and/or distribute water to the entire fish farm.

\section{Material and Methods}

\subsection{Study area}

The current work was carried out at Furnas Hydrobiology and Hatchery Station (20 40' S and $46^{\circ} 19^{\prime} \mathrm{W}$ ), Furnas Hydroelectric Plant (São José da Barra, MG, Brazil). The fish farm is located on the left bank of the Grande River, $2 \mathrm{~km}$ from the Furnas Reservoir, which provides water to the station's ponds. Continuous water flow provides a $5 \%$ daily exchange rate of the rearing volume.

Sampling sites were allocated from the reservoir where the water is harvested $\left(\mathrm{P}_{1}\right)$ for the fish farm up to the effluent outflow site $\left(\mathrm{P}_{6}\right)$ of the fish farm to the Grande River. Each fish pond has a separate water inflow system, but the water outflow pipe system of the tanks converges along the fish farm passing through sites $\left(\mathrm{P}_{3}, \mathrm{P}_{4}, \mathrm{P}_{5}\right.$ and $\left.\mathrm{P}_{6}\right)$ sampled within the fish farm. The fish farm has 34 ponds with area of $200 \mathrm{~m}^{2}, 2$ ponds with area of $1,000 \mathrm{~m}^{2}$, 160 ponds with area of $10 \mathrm{~m}^{2}, 9$ ponds with area varying between 150 and $400 \mathrm{~m}^{2}, 6$ ponds with approximately $2,500 \mathrm{~m}^{2}$, and a 5,670 $\mathrm{m}^{2}$ decantation pond $\left(\mathrm{P}_{5}\right)$. The ponds presented average depth varying from 1.00 to $5.00 \mathrm{~m}\left(\mathrm{P}_{5}\right)$. The sampling sites are ponds that receive and/or distribute water for the many different sections of the fish farm, except for site $\mathrm{P}_{5}$, which is a decantation pond. Water samples were collected from six sampling sites, as follows: site $1\left(\mathrm{P}_{1}\right)$, close to the impounding of source water (Furnas Reservoir); site $2\left(\mathrm{P}_{2}\right)$, a water distribution center to ponds and breeding ponds of the fish farm; site $3\left(\mathrm{P}_{3}\right)$, which receives effluent of the larva culture ponds; site $4\left(\mathrm{P}_{4}\right)$, which receives the effluent of the plankton production ponds; site $5\left(\mathrm{P}_{5}\right)$, in the lake which, besides having fish, receives water from the entire fish farm; and site $6\left(\mathrm{P}_{6}\right)$, where water is discharged from the fish farm directly onto the receiving water body (Grande river) without any prior treatment. Collections were undertaken every other day, during 20 consecutive days, during the rainy (February/02) and dry (August/02) seasons.

\subsection{Management}

Populations in the ponds comprised 'tilapia' (Oreochromis niloticus), 'trairão' (Hoplias larcedae), 'pintado' (Pseudoplastystoma corruscans), 'pacu' (Piaractus mesopotamicus), 'curimba' (Prochilodus lineatus), 'piapara' (Leporinus obtusidens), 'carpa' (Ciprinus carpio), 'dourado' (Salminus brasiliensis), and 'piau' (Leporinus friderici). The estimated total weight of fish reached 3 t. Continuous water flow comes from water at the Furnas Reservoir. Fish were fed a supplementary diet containing 15\% crude protein at the rate of $3 \%$ average live weight.

Fertilization in the ponds was done by chemical fertilizer $(25.6 \mathrm{~kg}$ of simple super phosphate and $48 \mathrm{~kg}$ of ammonium sulfate), and by organic fertilizer, adding $100 \mathrm{~kg}$ of pig dung in natura at approximately 7-day intervals, only to plankton ponds (near $\mathrm{P}_{4}$ ).

\subsection{Plankton}

At site $\mathrm{P}_{1}$, the plankton sample was taken in vertical hauls from the water column with totally filtered $45 \mathrm{~L}$ for the zooplankton and $25 \mathrm{~L}$ for the phytoplankton. At the other sites $\left(\mathrm{P}_{2}-\mathrm{P}_{6}\right)$, the plankton was sampled at the sub-surface with totally filtered $7.5 \mathrm{~L}$ by a Van Dorn bottle (2.5 L). Samples were obtained using $25 \mu \mathrm{m}$ and $55 \mu \mathrm{m}$-mesh nets, and preserved in lugol's solution and formalin (4\%), respectively, for quantitative and qualitative analysis of phytoplankton and zooplankton. Data were expressed in abundance percentage: specimens $\times 10^{3}$. $\mathrm{L}^{-1}$ for phytoplankton, and specimens. $\mathrm{L}^{-1}$ for zooplankton. Zooplankton organisms were counted in Sedgewick-Rafter 
Cell and examined under $100 \times$ magnification. Total sample count by sedimentation and density were calculated according to APHA (1989). Phytoplankton samples were identified and counted under an inverted microscope ( $400 \times$ magnification) using $10 \mathrm{~mL}$ Uthermöhl sedimentation chambers. Transects of each sample were counted to an error of less than 20\% (Lund et al., 1958). Frequency occurrence was also estimated for total phytoplankton and zooplankton organisms, divided into three categories: constant (50\% or above), common (between 10 and 50\%), or rare (between 1 and 10\%) (Sampaio et al., 2002).

\subsection{Hydrological variables}

Water samples were collected at a depth of $0.10 \mathrm{~m}$, with the exception of $\mathrm{P}_{5}$ at $2.5 \mathrm{~m}$, using a 5 L Van Dorn bottle. Nitrate and ammonium were determined according to Mackereth et al. (1978). Total phosphorus and orthophosphate were determined according to Murphy and Riley (1962). Temperature, dissolved oxygen and $\mathrm{pH}$ were measured in situ using a Horiba U-10 water quality checker.

\subsection{Statistical analysis}

The diversity index (H') (Shannon-Weaver), equitability and richness (total number of species) were used to analyze the diversity of phytoplankton and zooplankton between seasons and among sites (Odum and Barret, 2007). The ecological index values were obtained by estimating the mean values obtained for each season (dry and rainy) at each analyzed site $\left(\mathrm{P}_{1}\right.$ to $\left.\mathrm{P}_{6}\right)$. The criteria of Lobo and Leighton (1986) were applied to describe species dominance and abundance. Species were considered abundant when the number of specimens was higher than the mean density of all species; they were dominant when the density was higher than $50 \%$ of the total number of specimens. Pearson's correlation $(p<0.05)$ was used to find out the levels of significance of the relationship between groups of phytoplankton and zooplankton and environmental variables, by using the Statistics 6.0 program. Twoway ANOVA analysis was undertaken with physical and chemical variables to compare sites and seasons, and their interaction (Zar, 1996). Significance level was $\mathrm{p}<0.05$.

\section{Results}

Table 1 presents means of physical-chemical variables and inorganic nutrients during the dry and rainy seasons, with minimum and maximum values during the study period. No significant difference
( $p>0.05$ ) was observed among sites and between seasons for $\mathrm{pH}$. There was an increasing trend in inorganic nutrients starting at site $\mathrm{P}_{3}$, with the exception of nitrate $\left(\mathrm{P}_{2}\right)$. The mean concentrations of dissolved oxygen exceeded $4.3 \mathrm{mg} \cdot \mathrm{L}^{-1}$.

Rotifera species were dominant among the zooplankton community during the study period, with the exception of $\mathrm{P}_{1}$, where Cladocera was dominant during the dry season, and $\mathrm{P}_{2}$ and $\mathrm{P}_{3}$, where Copepoda were dominant during the dry and rainy season, respectively (Figure 1). Cladocera, namely Bosmina sp. (520 specimens. $\mathrm{L}^{-1}$ ), were predominant at $\mathrm{P}_{1}$ during the dry season; at $\mathrm{P}_{2}$ and $\mathrm{P}_{3}$ Copepoda were represented by Argyrodiaptomus furcatus (162 specimens. $\mathrm{L}^{-1}$ ) and Thermocyclops sp. nauplii (65 specimens. $\mathrm{L}^{-1}$ ), respectively, during the dry and rainy seasons. Although Cladocera were present at all sampled sites during the rainy season, they were absent during the dry season, appearing only at $\mathrm{P}_{1}, \mathrm{P}_{2}$ and $\mathrm{P}_{5}$. Copepoda were absent at $\mathrm{P}_{3}$ and $\mathrm{P}_{6}$ during the dry season, when Rotifera, namely Hexarthra intermedia and Bdelloidea species, made up more than $80 \%$ of organisms (Figure 1).

The zooplankton community was represented by 40 taxa, of which 30 belonged to Rotifera, 7 to Cladocera and 3 to Copepoda (Table 2). Rotifera species (Anuraeopsis navicula, Ascomorpha ecaudis, Brachionus calyciflorus, Keratella americana and Trichocerca similis) occurred at all sites throughout the study period, generally characterized as rare or common. Cladocera were constant only at site $\mathrm{P}_{1}$ associated directly with nitrate and $\mathrm{P}_{2}$ with ammonium during the dry season, represented by Bosmina longirostris and Bosmina sp., respectively (Table 2). The high frequency of Bosmina sp. during the rainy season at $\mathrm{P}_{1}$ and $\mathrm{P}_{2}$ was directly correlated with total phosphorus and orthophosphate (Tables 2 and 3). Daphnia sp., Mesocyclops sp., Conochilus unicornis, Trichocerca sp. were found only at $\mathrm{P}_{1}$ (Table 2); Asplanchna sp. and Brachionus cf. urceolaris were found at $\mathrm{P}_{2}$ when inverse relationships were observed with ammonium, total phosphorus and orthophosphate, during the rainy season (Tables 2 and 3). Cephalodella sp., Lecane cf. levistyla, and Lecane sp. were only found at $\mathrm{P}_{3}$ during the rainy season, associated with the highest mean concentrations of ammonium $\left(48.5 \mu \mathrm{g} . \mathrm{L}^{-1}\right)$ and temperature $\left(25.8{ }^{\circ} \mathrm{C}\right)$ observed during the study period (Tables 1 and 3 ). High concentrations of nitrate $\left(219.9 \mu \mathrm{g} . \mathrm{L}^{-1}\right)$ influenced the high abundance of Rotifera species during that period at site $\mathrm{P}_{3}$ (Tables 2 and 3; Figure 1). 
Table 1. Mean maximum and minimum (in parenthesis), temperature $\left({ }^{\circ} \mathrm{C}\right), \mathrm{pH}, \mathrm{DO}\left(\mathrm{mg} \cdot \mathrm{L}^{-1}\right)$ and nutrients $\left(\mu \mathrm{g} . \mathrm{L}^{-1}\right)$ during the dry $(\mathrm{D})$ and rainy $(\mathrm{R})$ seasons, at different sites $\left(\mathrm{P}_{1}-\mathrm{P}_{6}\right)$ of fish farm, and results of Two-way ANOVA among sites and seasons, where: Total Phospho. $=$ Total Phosphorus; Orthophospha. $=$ Orthophosphate; $\mathrm{DO}=$ dissolved oxygen; significant $=p<0.05$; not significant $p>0.05$.

\begin{tabular}{|c|c|c|c|c|c|c|c|c|c|c|c|c|c|}
\hline \multirow[t]{2}{*}{ Variables } & \multicolumn{2}{|c|}{$P_{1}$} & \multicolumn{2}{|c|}{$\mathrm{P}_{2}$} & \multicolumn{2}{|c|}{$\mathrm{P}_{3}$} & \multicolumn{2}{|c|}{$\mathrm{P}_{4}$} & \multicolumn{2}{|c|}{$P_{5}$} & \multicolumn{2}{|c|}{$\mathrm{P}_{6}$} & \multirow[t]{2}{*}{ ANOVA } \\
\hline & D & $\mathrm{R}$ & $D$ & $\mathrm{R}$ & D & $\mathrm{R}$ & D & $\mathrm{R}$ & D & $\mathrm{R}$ & $\mathrm{D}$ & $\mathrm{R}$ & \\
\hline Temperature & $\begin{array}{c}20.9 \\
(20-21)\end{array}$ & $\begin{array}{c}23.3 \\
(23-24)\end{array}$ & $\begin{array}{c}21.8 \\
(21-22)\end{array}$ & $\begin{array}{c}23.9 \\
(23-25\end{array}$ & $\begin{array}{c}22.3 \\
(21-23)\end{array}$ & $\begin{array}{c}25.8 \\
(25-27)\end{array}$ & $\begin{array}{c}22.2 \\
(22-24)\end{array}$ & $\begin{array}{c}25.8 \\
(25-28)\end{array}$ & $\begin{array}{c}22.8 \\
(22-25)\end{array}$ & $\begin{array}{c}26 \\
(25-28)\end{array}$ & $\begin{array}{c}23 \\
(22-25)\end{array}$ & $\begin{array}{c}26.5 \\
(25-29)\end{array}$ & $p<0.05$ \\
\hline $\mathrm{pH}$ & $\begin{array}{c}6.4 \\
(6.1-7)\end{array}$ & $\begin{array}{c}6.4 \\
(6.1-6.9)\end{array}$ & $\begin{array}{c}7 \\
(6-7.3)\end{array}$ & $\begin{array}{c}6.8 \\
(6.3-7.1)\end{array}$ & $\begin{array}{c}7 \\
(6-7.8)\end{array}$ & $\begin{array}{c}7 \\
(6.3-7.2)\end{array}$ & $\begin{array}{c}7.1 \\
(6.3-7.6)\end{array}$ & $\begin{array}{c}7 \\
(6.6-7.7)\end{array}$ & $\begin{array}{c}6.7 \\
(6.4-7.3)\end{array}$ & $\begin{array}{c}6.9 \\
(6.5-7.6)\end{array}$ & $\begin{array}{c}7.1 \\
(6.8-7.3)\end{array}$ & $\begin{array}{c}7.1 \\
(6.8-7.4)\end{array}$ & $p>0.05$ \\
\hline DO & $\begin{array}{c}5.6 \\
(4.2-10.2)\end{array}$ & $\begin{array}{c}5.6 \\
(4.8-7.1)\end{array}$ & $\begin{array}{c}7.8 \\
(6.5-8.0)\end{array}$ & $\begin{array}{c}7.5 \\
(6.5-7.7)\end{array}$ & $\begin{array}{c}7.3 \\
(6.6-7.5)\end{array}$ & $\begin{array}{c}6.8 \\
(4.8-7.3)\end{array}$ & $\begin{array}{c}7.3 \\
(7.0-7.6)\end{array}$ & $\begin{array}{c}6.8 \\
4.5-8.2)\end{array}$ & $\begin{array}{c}4.3 \\
(2.5-5.6)\end{array}$ & $\begin{array}{c}6.5 \\
(3.7-7.8)\end{array}$ & $\begin{array}{c}7.1 \\
(6.8-7.6)\end{array}$ & $\begin{array}{c}7.1 \\
(3.2-7.6)\end{array}$ & $p<0.05$ \\
\hline Ammonium & $\begin{array}{c}7.1 \\
(2-18)\end{array}$ & $\begin{array}{c}17 \\
(3-110)\end{array}$ & $\begin{array}{c}5.1 \\
(1-10)\end{array}$ & $\begin{array}{c}8.4 \\
(2-16)\end{array}$ & $\begin{array}{c}154.6 \\
(16-1,397)\end{array}$ & $\begin{array}{c}48.5 \\
(5-103)\end{array}$ & $\begin{array}{c}70.3 \\
(4-355)\end{array}$ & $\begin{array}{c}42.6 \\
(12-101)\end{array}$ & $\begin{array}{c}141.3 \\
(9-241)\end{array}$ & $\begin{array}{c}42.8 \\
(5-95)\end{array}$ & $\begin{array}{c}138.2 \\
(44-245)\end{array}$ & $\begin{array}{c}26.9 \\
(8-86)\end{array}$ & $p<0.05$ \\
\hline Nitrate & $\begin{array}{c}136.1 \\
(100-167)\end{array}$ & $\begin{array}{c}237.2 \\
(166-292)\end{array}$ & $\begin{array}{c}144.3 \\
(106-190)\end{array}$ & $\begin{array}{c}257.2 \\
(178-299)\end{array}$ & $\begin{array}{c}149.6 \\
(77-216)\end{array}$ & $\begin{array}{c}219.9 \\
(154-282)\end{array}$ & $\begin{array}{c}142.6 \\
(103-179)\end{array}$ & $\begin{array}{c}190.4 \\
(103-253)\end{array}$ & $\begin{array}{c}130.4 \\
(101-166)\end{array}$ & $\begin{array}{c}157.2 \\
(88-221)\end{array}$ & $\begin{array}{c}126.9 \\
(100-149)\end{array}$ & $\begin{array}{c}150.1 \\
(82-209)\end{array}$ & $p<0.05$ \\
\hline Total Phospho. & $\begin{array}{c}11.4 \\
(6-16)\end{array}$ & $\begin{array}{c}12.6 \\
(3-37)\end{array}$ & $\begin{array}{c}10.9 \\
(5-20)\end{array}$ & $\begin{array}{c}9.7 \\
(4-23)\end{array}$ & $\begin{array}{c}61.7 \\
(18-186)\end{array}$ & $\begin{array}{c}99.5 \\
(22-831)\end{array}$ & $\begin{array}{c}40.6 \\
(24-77)\end{array}$ & $\begin{array}{c}103.2 \\
(25-753)\end{array}$ & $\begin{array}{c}76.4 \\
(57-100)\end{array}$ & $\begin{array}{c}54.1 \\
(30-99)\end{array}$ & $\begin{array}{c}77.1 \\
(55-94)\end{array}$ & $\begin{array}{c}53.7 \\
(27-88)\end{array}$ & $p<0.05$ \\
\hline Orthophospha. & $\begin{array}{c}6 \\
(2-10) \\
\end{array}$ & $\begin{array}{c}3.3 \\
(1-8) \\
\end{array}$ & $\begin{array}{c}5.7 \\
(3-9) \\
\end{array}$ & $\begin{array}{c}3.2 \\
(1-8) \\
\end{array}$ & $\begin{array}{c}44.7 \\
(11-178)\end{array}$ & $\begin{array}{c}64 \\
(9-595) \\
\end{array}$ & $\begin{array}{c}19.4 \\
(7-64)\end{array}$ & $\begin{array}{c}56.8 \\
(5-491) \\
\end{array}$ & $\begin{array}{c}13.0 \\
(8-18)\end{array}$ & $\begin{array}{c}7.5 \\
(2-29) \\
\end{array}$ & $\begin{array}{c}11.4 \\
(7-18)\end{array}$ & $\begin{array}{c}6.6 \\
(1-23) \\
\end{array}$ & $p<0.05$ \\
\hline
\end{tabular}
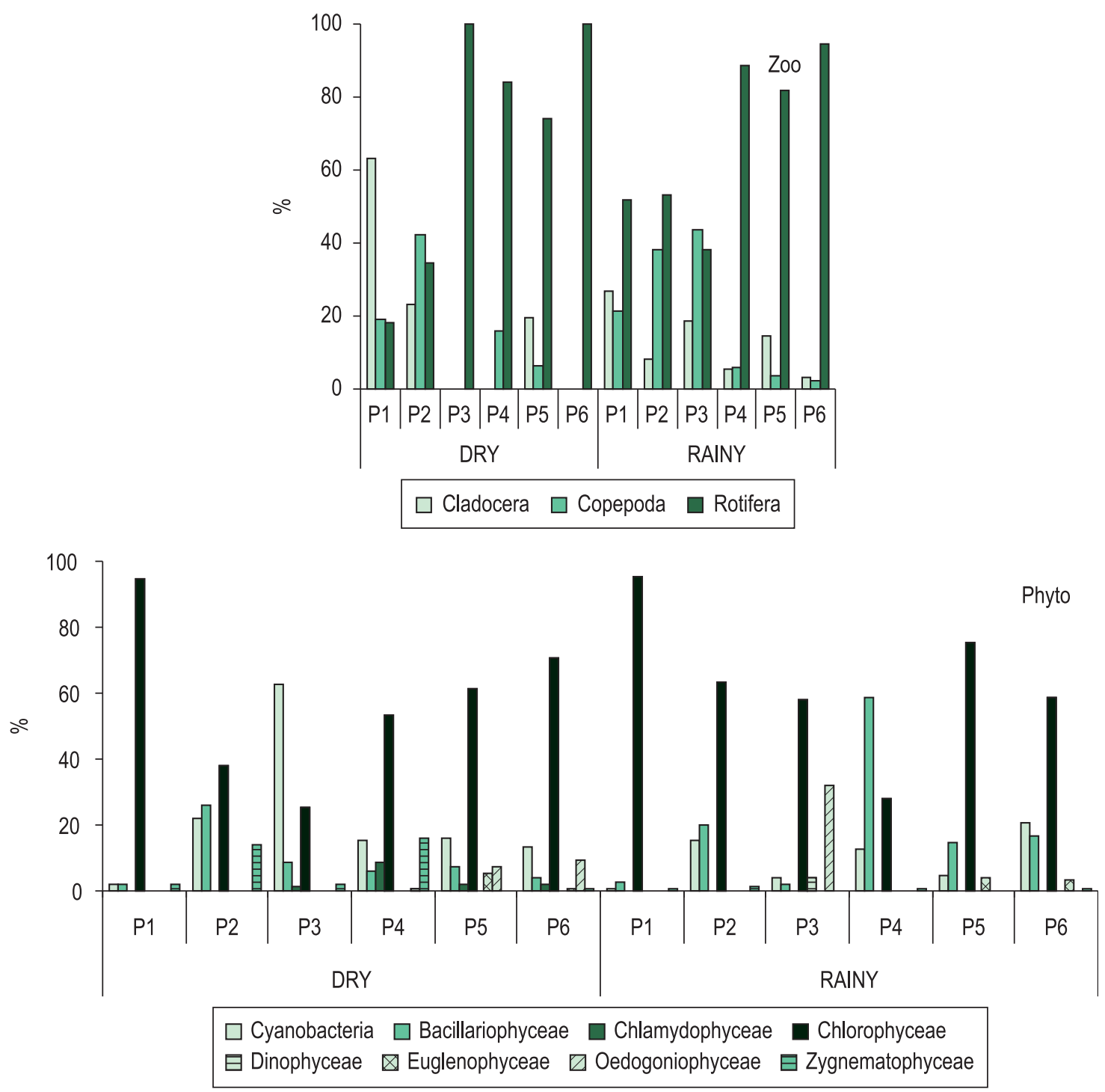

Figure 1. Relative abundance (\%) of zooplankton (Zoo) and phytoplankton (Phyto) at the sites $\left(\mathrm{P}_{1}-\mathrm{P}_{6}\right)$ during the dry and rainy seasons. 
Table 2. Specific composition (+, -), frequency of occurrence (F) and abundance of zooplankton taxa in the dry (D) and rainy $(\mathrm{R})$ seasons at the sites $\left(\mathrm{P}_{1}-\mathrm{P}_{6}\right)$, where: $+=$ presence; $-=$ absence; $\mathrm{A}=$ abundant; $\mathrm{d}=$ dominant; $\mathrm{C}=$ constant; $\mathrm{c}=$ common and $\mathrm{r}=$ rare.

\begin{tabular}{|c|c|c|c|c|c|c|c|c|c|c|c|c|c|c|c|c|c|c|c|c|c|c|c|}
\hline \multirow[t]{2}{*}{ Taxa } & \multicolumn{4}{|c|}{$P_{1}$} & \multicolumn{4}{|c|}{$\mathrm{P}_{2}$} & \multicolumn{4}{|c|}{$\mathrm{P}_{3}$} & \multicolumn{4}{|c|}{$\mathrm{P}_{4}$} & \multicolumn{4}{|c|}{$P_{5}$} & \multicolumn{3}{|c|}{$P_{6}$} \\
\hline & $\mathrm{D}$ & $\mathrm{F}$ & $\mathrm{R}$ & $\mathrm{F}$ & $\mathrm{D}$ & $\mathrm{F}$ & $\mathrm{R}$ & $\mathrm{F}$ & $\mathrm{D}$ & $\mathrm{F}$ & $\mathrm{R}$ & $\mathrm{F}$ & D & $\mathrm{F}$ & $\mathrm{R}$ & $\mathrm{F}$ & $\mathrm{D}$ & $\mathrm{F}$ & $\mathrm{R}$ & $\mathrm{F}$ & $\mathrm{D}$ & $\mathrm{F} R$ & R F \\
\hline \multicolumn{24}{|l|}{ Cladocera } \\
\hline Bosmina longirostris Muller, 1785 & + & C & + & r & - & & - & & - & & + & $r$ & - & & - & & - & & - & & - & & $+r$ \\
\hline Bosmina sp. & + & $r$ & + & $c$ & + & C & + & c & - & & - & & - & & - & & - & & + & r & - & & $+r$ \\
\hline Daphnia gessneri Herbst, 1967 & - & & + & $r$ & - & & - & & - & & + & $r$ & - & & - & & - & & - & & - & & - \\
\hline Daphnia sp. & + & $r$ & + & r & - & & - & & - & & - & & - & & - & & - & & - & & - & & - \\
\hline Diaphanosoma birge Korinek, 1981 & + & r & + & r & - & & - & & - & & - & & - & & + & r & + & r & + & $r$ & - & & - \\
\hline Moina minuta Hansen, 1899 & + & $r$ & + & r & - & & - & & - & & + & $c$ & - & & - & & + & r & - & & - & & - \\
\hline Moina sp. & + & $r$ & + & $r$ & - & & + & $r$ & - & & + & $r$ & - & & - & & - & & - & & - & & $+r$ \\
\hline \multicolumn{24}{|l|}{ Copepoda } \\
\hline Argyrodiaptomus furcatus Sars, 1901 & + & $r$ & + & $c$ & + & c & + & $r$ & 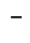 & & - & & - & & + & $r$ & + & $r$ & + & $r$ & - & & - \\
\hline copepodid & - & & + & r & - & & + & $r$ & - & & - & & - & & - & & - & & - & & - & & d c c a a a \\
\hline nauplii & - & & + & $r$ & - & & + & c & - & & + & $r$ & - & & + & r & - & & + & $r$ & - & & - \\
\hline Mesocyclops sp. & - & & + & $r$ & - & & - & & - & & - & & - & & - & & - & & - & & - & & - \\
\hline Thermocyclops sp. & - & & + & $r$ & - & & + & $r$ & 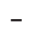 & & + & $r$ & - & & - & & - & & + & $r$ & - & & $+r$ \\
\hline copepodid & - & & + & $r$ & - & & - & & - & & - & & - & & - & & - & & + & $r$ & - & & - \\
\hline nauplii & - & & - & & - & & + & $r$ & - & & + & c & - & & + & $r$ & - & & + & $r$ & - & & + \\
\hline
\end{tabular}

Rotifera

Anuraeopsis fissa (Gosse, 1851)

Anuraeopsis navicula (Rousselet, 1911)

Ascomorpha agilis Zacharias, 1893

Ascomorpha ecaudis Perty, 1850

Ascomorpha saltans Kolisko, 1938

Asplanchna sp.

Bdelloidea not determined

Brachionus calyciflorus Pallas, 1766

Brachionus falcatus Zacharias, 1898

Brachionus cf. urceolaris Müller, 1773

Cephalodella sp.

Collotheca sp.

Conochlius unicornis Rousselet, 1892

Epiphanes sp.

Euchlanis sp.

Filinia longiseta Ehrenberg, 1834

Gastropus sp.

Hexarthra intermedia Wiszniewski, 1929

Keratella americana Carlin, 1943

Keratella tropica (Apstein,1907)

Lecane cf. levistyla (Olofsson, 1917)

Lecane M lunaris Ehrenberg, 1832

Lecane sp.

Lepadella patella (Müller, 1786)

Polyarthra vulgaris (Carlin, 1943)

Ptygura sp.

Trichocerca cylindrica (Imhof, 1891)

Trichocerca pusilla (Lauterborn, 1898)

Trichocerca similis (Wierzejski, 1893)

Trichocerca sp. 
Table 3. Pearson's correlation between environmental variables and phytoplankton and zooplankton groups in the dry $(\mathrm{D})$ and rainy $(\mathrm{R})$ seasons at the sites $\left(\mathrm{P}_{1}-\mathrm{P}_{6}\right)$, where: $\mathrm{TP}=$ total phosphorus; Ortho. = orthophosphate; Temp.= temperature; Amm. = ammonium; and $\mathrm{p}<0.05$.

\begin{tabular}{|c|c|c|c|c|c|c|c|c|c|c|c|c|}
\hline \multirow[t]{2}{*}{ Variables } & \multicolumn{2}{|c|}{$P_{1}$} & \multicolumn{2}{|c|}{$\mathrm{P}_{2}$} & \multicolumn{2}{|c|}{$\mathrm{P}_{3}$} & \multicolumn{2}{|c|}{$\mathrm{P}_{4}$} & \multicolumn{2}{|c|}{$P_{5}$} & \multicolumn{2}{|c|}{$P_{6}$} \\
\hline & $\mathrm{D}$ & $\mathrm{R}$ & $\mathrm{D}$ & $\mathrm{R}$ & $\mathrm{D}$ & $\mathrm{R}$ & $\mathrm{D}$ & $\mathrm{R}$ & $\mathrm{D}$ & $\mathrm{R}$ & $\mathrm{D}$ & $\mathrm{R}$ \\
\hline \multicolumn{13}{|l|}{ Phytoplankton } \\
\hline Bacillariophyceae and nitrate & 0.11 & - & 0.55 & - & - & 0.78 & - & 0.23 & 0.40 & 0.37 & - & - \\
\hline Bacillariophyceae and Temp.. & - & - & 0.39 & - & - & - & - & -0.09 & - & - & - & - \\
\hline Bacillariophyceae and Ortho. & _ & - & - & 1.00 & 0.65 & - & _- & 0.27 & _- & _- & _- & 0.32 \\
\hline Bacillariophyceae and Amm. & _ & _ & _ & _- & _ & _- & -0.68 & 0.25 & _ & _- & 0.25 & 0.64 \\
\hline Bacillariophyceae and TP & 0.90 & - & - & 1.00 & 0.48 & - & - & 0.45 & _ & 0.30 & - & - \\
\hline Chlorophyceae and nitrate & -0.74 & 0.31 & - & - & - & 0.42 & - & 0.55 & - & - & 0.37 & 0.40 \\
\hline Chlorophyceae and Ortho. & 0.54 & 0.47 & - & 1.00 & 0.89 & 0.39 & - & - & - & - & - & - \\
\hline Chlorophyceae and TP & - & - & - & 1.00 & 0.96 & & - & - & - & 0.30 & - & - \\
\hline Cyanobacteria and nitrate & _- & 0.61 & 0.37 & _- & 0.68 & & 0.20 & _- & _ & 0.37 & _- & _- \\
\hline Cyanobacteria and Temp. & - & 0.51 & - & - & 0.80 & 0.61 & - & - & _ & _- & - & - \\
\hline Cyanobacteria and Ortho. & - & 0.93 & - & 1.00 & -0.54 & - & - & - & - & - & - & - \\
\hline Cyanobacteria and Amm. & - & 0.86 & _ & _- & 0.62 & _ & - & - & _ & _- & _ & 0.40 \\
\hline Cyanobacteria and TP & 0.90 & 0.67 & -0.33 & 1.00 & -0.49 & - & - & - & - & - & - & - \\
\hline \multicolumn{13}{|l|}{ Zooplankton } \\
\hline Cladocera and nitrate & 0.08 & -0.01 & - & -0.48 & - & - & - & - & - & - & - & -0.40 \\
\hline Cladocera and Temp. & - & -0.46 & - & -0.54 & - & 0.73 & - & - & - & - & - & - \\
\hline Cladocera and Ortho. & - & 0.12 & - & 0.15 & - & - & - & - & - & - & - & - \\
\hline Cladocera and Amm. & _- & -0.01 & 0.54 & -0.34 & _ & _- & _- & _- & _- & _- & _ & 0.78 \\
\hline Cladocera and TP & - & 0.09 & - & 0.14 & - & 0.38 & - & - & - & _ & - & 0.45 \\
\hline Copepoda and nitrate & 0.20 & - & 0.46 & - & _ & - & - & 0.43 & _- & - & _ & 0.41 \\
\hline Copepoda and Ortho. & - & - & - & - & - & - & - & - & - & - & - & 0.85 \\
\hline Copepoda and TP & - & 0.38 & - & - & - & - & - & - & - & - & - & 0.44 \\
\hline Rotifera and nitrate & 0.19 & _- & _ & _- & _- & 0.16 & 0.25 & _- & 0.67 & _ & _ & _- \\
\hline Rotifera and Temp. & - & - & -0.78 & -0.37 & - & 0.50 & 0.70 & - & - & - & 0.50 & - \\
\hline Rotifera and Ortho. & -0.65 & 0.64 & -0.30 & -0.30 & - & -0.24 & _ & - & - & - & -0.56 & - \\
\hline Rotifera and Amm. & - & - & 0.31 & -0.04 & - & 0.12 & - & 0.70 & _ & _ & -0.61 & - \\
\hline Rotifera and TP & - & 0.38 & - & -0.35 & - & - & - & - & - & - & - & 0.44 \\
\hline
\end{tabular}

Among the phytoplankton community, the dominant species were: Phromidium sp. at site $\mathrm{P}_{3}$, correlated with nitrate, ammonium and temperature during the dry season; Synedra sp. at site $\mathrm{P}_{3}$, correlated with nitrate during the rainy season; and Chlorella sp. at site $\mathrm{P}_{1}$, correlated with nitrate and orthophosphate during rainy season (Tables 3 and 4). Among the zooplankton community, only copepod Argyrodiaptomus furcatus was dominant at site $\mathrm{P}_{6}$ during the rainy season, as a function of orthophosphate (Tables 2 and 3).

Chlorophyceae were abundant at all sites during both seasons, except at $\mathrm{P}_{3}$ during the dry season and $\mathrm{P}_{4}$ during the rainy season (Figure 1). Predominance of Cyanobacteria occurred during the dry period due to the high density of Microcystis sp. $\left(285\right.$ specimens $\left.\times 10^{3} \cdot \mathrm{L}^{-1}\right)($ Table 3) and Bacillariophyceae at $\mathrm{P}_{4}$ during the rainy season due to Synedra sp. $\left(1,465\right.$ specimens $\left.\times 10^{3} \cdot \mathrm{L}^{-1}\right)$. The dominance of Cyanobacteria at site $\mathrm{P}_{3}$ during the dry season coincided with the high mean levels of ammonium (154.6 $\left.\mu \mathrm{g} . \mathrm{L}^{-1}\right)$, nitrate $\left(149.6 \mu \mathrm{g} . \mathrm{L}^{-1}\right)$ and temperature $\left(22.3{ }^{\circ} \mathrm{C}\right.$ ) (Tables 1 and 3; Figure 1). The high abundance of Bacillariophyceae at $\mathrm{P}_{4}$ during the rainy season was related to nutrients, especially total phosphorus (Table 3; Figure 1).

Fourteen algae species occurred at all sampled sites (Table 4). However, only Chlorella sp. and Crucigena sp. were present in both seasons at all sites; Chlorella sp. occurred generally between 10 and $50 \%$ of the total number of organisms and Crucigena sp. occurred below 10\% (Table 4). As a rule, Zygnematophyceae species were less than $10 \%$ of total algae, with the exception of Staurastrum sp. at site $\mathrm{P}_{6}$ during the dry season (Table 4). Euglenophyceae species occurred only at sites $\mathrm{P}_{4}, \mathrm{P}_{5}$ and $\mathrm{P}_{6}$ in both seasons (Table 4; Figure 1) in which nitrate rates were above $126 \mu \mathrm{g} . \mathrm{L}^{-1}$ (Table 1).

The ecological indices species richness, Shannon's diversity and species equitability generally showed the highest values for the phytoplankton community 
Table 4. Species composition $(+,-)$, frequency of occurrence (F) and abundance of phytoplankton taxa in the dry (D) and rainy $(R)$ seasons at the sites $\left(\mathrm{P}_{1}-\mathrm{P}_{6}\right)$, where: $+=$ presence; $-=$ absence; $\mathrm{A}=$ abundant; $\mathrm{d}=$ dominant; $\mathrm{C}=$ constant; $\mathrm{c}=$ common and $\mathrm{r}=$ rare.

\begin{tabular}{|c|c|c|c|c|c|c|c|c|c|c|c|c|c|c|c|c|c|c|c|c|c|c|c|c|}
\hline \multirow[t]{2}{*}{ Taxa } & \multicolumn{4}{|c|}{$P_{1}$} & \multicolumn{4}{|c|}{$\mathrm{P}_{2}$} & \multicolumn{4}{|c|}{$\mathrm{P}_{3}$} & \multicolumn{4}{|c|}{$\mathrm{P}_{4}$} & & $\mathrm{P}$ & & & & & 6 & \\
\hline & $\mathrm{D}$ & $\mathrm{F}$ & $\mathrm{R}$ & $\mathrm{F}$ & $D$ & $\mathrm{~F}$ & $\mathrm{R}$ & $\mathrm{F}$ & $\mathrm{D}$ & $\mathrm{F}$ & $\mathrm{R}$ & $\mathrm{F}$ & D & $\mathrm{F}$ & $\mathrm{R}$ & $\mathrm{F}$ & $\mathrm{D}$ & $\mathrm{F}$ & $\mathrm{R}$ & $\mathrm{F}$ & $D$ & $\mathrm{~F}$ & $\mathrm{R}$ & $\mathrm{F}$ \\
\hline Cyanobacteria & & & & & & & & & & & & & & & & & & & & & & & & \\
\hline Anabaena sp. & + & $r$ & - & & + & $r$ & - & & + & $r$ & - & & + & $r$ & + & $r$ & + & $r$ & + & $r$ & + & r & + & $r$ \\
\hline Aphanothece sp. & + & $r$ & - & & - & & - & & - & & - & & - & & - & & + & r & - & & - & & - & \\
\hline Borzia sp. & - & & - & & - & & - & & - & & - & & + & $\mathrm{r}$ & - & & - & & - & & + & $\mathrm{r}$ & - & \\
\hline Chroococcus sp. & - & & - & & - & & - & & - & & - & & - & & - & & + & r & - & & - & & - & \\
\hline Lyngbya sp. & + & $r$ & - & & - & & - & & - & & + & $r$ & + & $r$ & + & $r$ & + & $r$ & + & $r$ & - & & + & $r$ \\
\hline Merismopedia sp. & - & & - & & - & & - & & - & & - & & + & $\mathrm{r}$ & - & & + & $\mathrm{r}$ & + & $\mathrm{r}$ & + & $r$ & + & $\mathrm{r}$ \\
\hline Microcystis sp. & - & & - & & - & & - & & + & C & + & $r$ & + & $r$ & + & $r$ & + & $r$ & + & $r$ & + & $\mathrm{C}$ & + & $r$ \\
\hline Oscillatoria sp. & - & & + & $r$ & - & & + & $r$ & + & $r$ & + & r & + & r & + & $r$ & - & & + & $r$ & + & $r$ & - & \\
\hline Phormidium sp. & + & $r$ & - & & - & & - & & d & c & - & & - & & - & & + & $\mathrm{r}$ & + & $\mathrm{r}$ & + & $r$ & - & \\
\hline Spirullina sp. & - & & - & & - & & - & & - & & - & & + & r & - & & - & & + & $r$ & - & & - & \\
\hline Bacillariophyceae & & & & & & & & & & & & & & & & & & & & & & & & \\
\hline Amphipleura sp. & - & & - & & - & & - & & - & & - & & - & & - & & - & & + & $r$ & - & & - & \\
\hline Asterionella sp. & - & & - & & - & & - & & - & & - & & + & r & + & $r$ & + & r & - & & + & $r$ & - & \\
\hline Aulacoseira sp. & + & $r$ & + & $\mathrm{r}$ & - & & + & & + & $r$ & + & $r$ & + & $\mathrm{r}$ & + & $\mathrm{r}$ & + & $\mathrm{r}$ & + & $\mathrm{r}$ & + & $r$ & + & $\mathrm{r}$ \\
\hline Cyclotella sp. & + & $r$ & - & & - & & + & $r$ & - & & - & & - & & - & & + & $r$ & + & $r$ & - & & - & \\
\hline Cymbella sp. & - & & + & $\mathrm{r}$ & + & c & + & $\mathrm{r}$ & + & $r$ & + & $\mathrm{r}$ & + & $\mathrm{r}$ & + & $\mathrm{r}$ & - & & + & $\mathrm{r}$ & + & $\mathrm{r}$ & + & $\mathrm{r}$ \\
\hline Diatomella sp. & - & & - & & - & & - & & - & & - & & + & $r$ & - & & - & & - & & + & $r$ & - & \\
\hline Frustulia sp. & - & & - & & - & & + & $r$ & + & $r$ & - & & + & r & + & r & + & r & + & $r$ & + & $r$ & + & $r$ \\
\hline Gomphonema sp. & - & & - & & + & r & - & & + & $r$ & - & & - & & - & & - & & - & & - & & - & \\
\hline Melosira sp. & + & $r$ & - & & - & & - & & - & & - & & + & r & + & r & + & r & + & $r$ & - & & + & $r$ \\
\hline Surirella sp. & - & & - & & - & & - & & - & & + & $r$ & - & & + & r & + & $r$ & - & & + & $r$ & - & \\
\hline Synedra sp. & - & & + & $r$ & - & & + & $r$ & - & & $d$ & c & - & & + & C & - & & + & $r$ & - & & + & $r$ \\
\hline Chlamydophyceae & & & & & & & & & & & & & & & & & & & & & & & & \\
\hline Pandorina sp. & - & & - & & - & & - & & + & $r$ & - & & + & $r$ & - & & + & r & - & & + & $r$ & - & \\
\hline Chlorophyceae & & & & & & & & & & & & & & & & & & & & & & & & \\
\hline Ankistrodesmus sp. & + & $r$ & - & & - & & - & & + & $r$ & - & & - & & + & r & + & r & + & $r$ & - & & - & \\
\hline Botryococcus sp. & + & $r$ & - & & + & $r$ & - & & + & $r$ & - & & + & $r$ & + & r & + & $r$ & + & $r$ & + & $r$ & + & $r$ \\
\hline Chlorella sp. & + & C & $d$ & C & + & c & + & C & + & $r$ & + & c & + & C & + & c & + & c & + & c & + & $r$ & + & c \\
\hline Closteriopsis sp. & + & $r$ & + & r & + & c & + & r & - & & - & & + & $r$ & + & c & - & & + & c & + & $r$ & + & c \\
\hline Coelastrum reticulatum (Dangeard) & - & & - & & - & & - & & - & & + & r & + & r & + & r & + & r & - & & + & $\mathrm{r}$ & - & \\
\hline Coelastrum sp. & + & $r$ & + & c & + & r & + & r & + & $r$ & - & & + & c & - & & + & c & + & c & + & c & + & $r$ \\
\hline Crucigenia sp. & + & $r$ & + & $r$ & + & $r$ & + & $r$ & + & $r$ & + & $r$ & + & $r$ & + & r & + & r & + & $r$ & + & $r$ & + & $r$ \\
\hline Dictyosphaerium sp. & + & $r$ & + & r & - & & - & & + & $r$ & + & $r$ & + & r & + & r & + & r & + & r & + & $\mathrm{r}$ & + & $r$ \\
\hline Eutetramorus sp. & + & $r$ & - & & - & & + & $r$ & + & $r$ & + & $r$ & + & r & + & r & + & r & + & $r$ & + & $r$ & + & $r$ \\
\hline Franceia sp. & - & & - & & - & & - & & + & $r$ & - & & - & & - & & - & & - & & + & $\mathrm{r}$ & - & \\
\hline Kirchneriella sp. & + & $r$ & - & & - & & + & r & + & $r$ & + & $r$ & + & r & + & r & + & r & + & r & + & $r$ & + & r \\
\hline Monoraphidium sp. & + & $r$ & + & $r$ & - & & + & r & + & $r$ & + & $r$ & + & r & + & r & + & r & + & $\mathrm{r}$ & + & $r$ & + & $r$ \\
\hline Pediastrum duplex Meyen & - & & - & & - & & + & $r$ & - & & - & & - & & - & & + & r & - & & + & $r$ & - & \\
\hline Pediastrum simplex (Meyen) & - & & - & & - & & - & & - & & + & $r$ & - & & - & & + & r & - & & + & $r$ & - & \\
\hline Pediastrum sp. Meyen & + & $r$ & + & r & - & & + & r & + & r & - & & + & r & + & r & - & & + & r & - & & + & $\mathrm{r}$ \\
\hline Scenedesmus ecornis (Ehrenberg) Chodat & + & $r$ & + & r & - & & - & & + & $r$ & + & $r$ & - & & + & r & + & r & + & $r$ & - & & + & $r$ \\
\hline Scenedesmus quadricauda (Turpin) Brébison & + & $r$ & + & r & + & r & + & & + & r & + & r & + & $r$ & - & & + & r & + & $\mathrm{r}$ & + & $c$ & + & $\mathrm{r}$ \\
\hline Tetraëdron sp. & - & & + & r & - & & - & & + & r & - & & + & r & + & r & - & & - & & + & $r$ & + & $\mathrm{r}$ \\
\hline Tetrastrum triangulare (Chodat) Komárek & + & $r$ & - & & - & & - & & - & & - & & - & & - & & + & r & + & r & - & & - & \\
\hline Dinophyceae & & & & & & & & & & & & & & & & & & & & & & & & \\
\hline Ceratium sp. & - & & - & & - & & - & & - & & + & $\mathrm{r}$ & - & & - & & - & & - & & - & & - & \\
\hline Euglenophyceae & & & & & & & & & & & & & & & & & & & & & & & & \\
\hline Euglena sp. & - & & - & & - & & - & & - & & - & & - & & - & & + & $\mathrm{r}$ & + & $\mathrm{r}$ & + & $r$ & - & \\
\hline Phacus sp. & - & & - & & - & & - & & - & & - & & - & & + & r & + & r & + & $\mathrm{r}$ & - & & + & $\mathrm{r}$ \\
\hline Trachelomonas sp. & - & & - & & - & & - & & - & & - & & - & & - & & - & & + & r & - & & - & \\
\hline Oedogoniophyceae & & & & & & & & & & & & & & & & & & & & & & & & \\
\hline Bulbochaete sp. & - & & - & & - & & - & & - & & + & c & - & & - & & - & & + & $r$ & - & $\mathrm{r}$ & - & \\
\hline Oedogonium sp. & - & & - & & - & & - & & - & & - & & + & $r$ & - & & + & r & - & & + & c & - & \\
\hline Zygnematophyceae & & & & & & & & & & & & & & & & & & & & & & & & \\
\hline Closterium sp. & - & & - & & + & $r$ & - & & + & $r$ & - & & + & $r$ & - & & + & r & - & & - & & - & \\
\hline Cosmarium sp. & - & & - & & - & & - & & - & & - & & + & $r$ & + & $r$ & - & & - & & - & & - & \\
\hline Micrasterias sp. & - & & - & & - & & - & & + & $r$ & - & & - & & - & & - & & - & & - & & - & \\
\hline Mougeotia sp. & - & & - & & - & & - & & - & & - & & + & r & - & & - & & - & & - & & - & \\
\hline Staurastrum sp. & + & $r$ & + & $r$ & + & $r$ & + & $r$ & + & $r$ & - & & + & $r$ & + & $r$ & + & r & + & $r$ & + & $c$ & + & $r$ \\
\hline Zygnema sp. & - & & - & & - & & - & & - & & - & & + & $r$ & - & & - & & - & & - & & - & \\
\hline
\end{tabular}


during the dry season. Lower values for zooplankton occurred during the dry season, with mean species richness of 7.9 and 0.56 for diversity, when compared with the rainy season (20.5 and 1.08, respectively). The values of species richness for zooplankton and diversity indices were highest during the rainy season. The equitability and diversity values for the zooplankton community decreased from site $\mathrm{P}_{1}$ to $\mathrm{P}_{6}$, with a slight increase at $\mathrm{P}_{5}$ during the dry season (Figure 2).

\section{Discussion}

Due to the management employed at the fish farm with the addition of feed and fertilizers to increase productivity, there were high levels of nutrients in the water, which directly interfered in
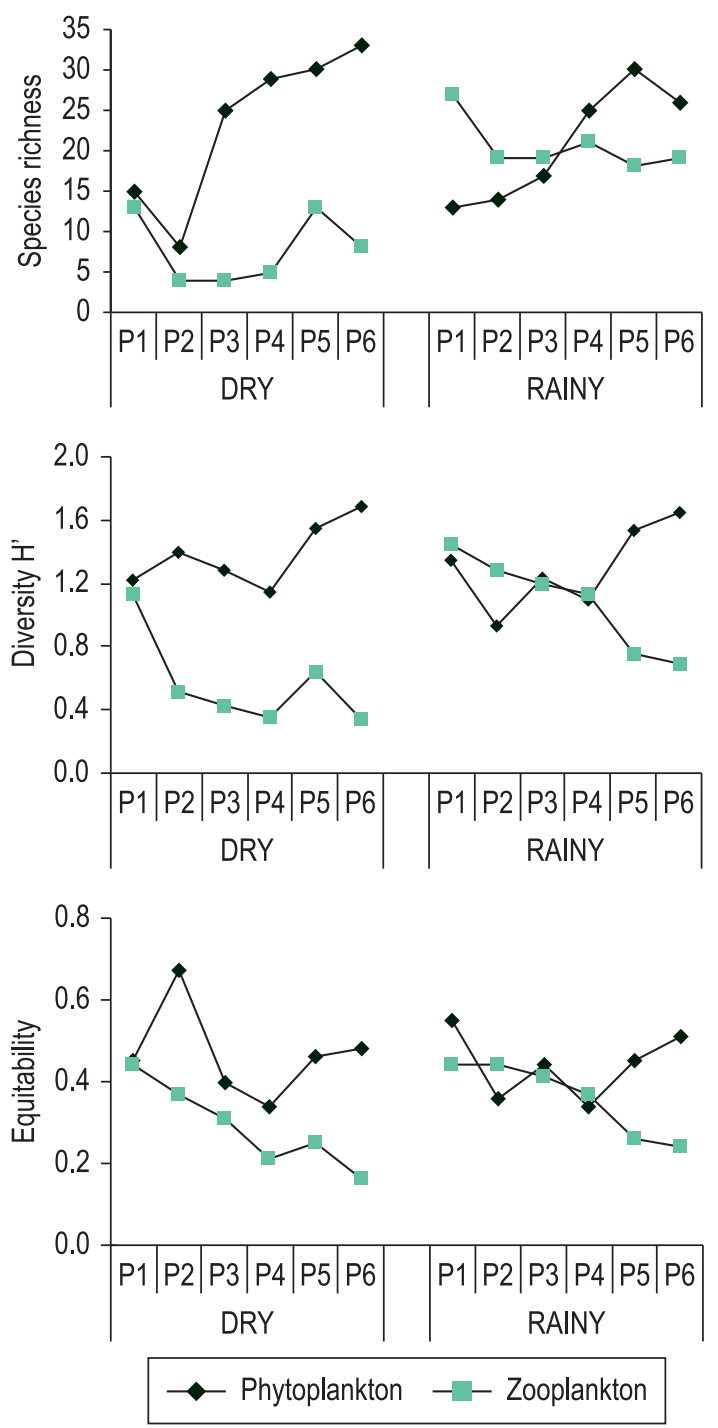

Figure 2. Ecological indices (richness, diversity and species equitability) of zooplankton and phytoplankton during the dry and rainy seasons at the different sites $\left(\mathrm{P}_{1}-\mathrm{P}_{6}\right)$ in the fish farm. the plankton community. Sites $\mathrm{P}_{1}$ and $\mathrm{P}_{2}$ (source water), which do not receive the allochthonous matter of the fish farm, showed lower nutrient levels than sites located within the fish farm $\left(\mathrm{P}_{3}\right.$ to $\left.\mathrm{P}_{6}\right)$; the sites near the plankton production ponds $\left(\mathrm{P}_{3}\right.$ and $\mathrm{P}_{4}$ ) generally showed the highest concentration of nutrients due to the organic matter (pig dung) resulting from the fertilization of these ponds.

The role of fertilizers in increasing fish production has been emphasized, and fertilizers with ammonium sulfate stimulate phytoplankton growth and zooplankton production. The combined action of nitrogen and phosphorus stimulates high production as dominance of Cyanobacteria in the fish farm (Dhawan and Laur, 2002).

The influence of fertilizers in the plankton community was observed in this study where Cyanobacteria was dominant at site $\mathrm{P}_{3}$, and only Rotifera were observed at that site during the dry season, while Cyanobacteria showed a positive correlation with ammonium, nitrate and temperature, and an inverse correlation with total phosphorus and orthophosphate. The dominance of Cyanobacteria only at $\mathrm{P}_{3}$ during the dry season was due to the presence of Microcystis sp. It is well known that the N:P ratio is also an important factor for the dominance of Cyanobacteria (Imai et al., 2009). Water temperature is another possibly important controlling factor for the seasonal succession of Microcystis species. The preferred temperature range of Microcystis species is from 24 to $34^{\circ} \mathrm{C}$; changing water temperature is one important factor controlling the composition of Cyanobacteria (Imai et al., 2009).

Phytoplankton growth and development are mainly steered by available solar energy input, hydrodynamic forces such as stratification and mixing in the resulting levels of nitrogen and phosphorus (Chellappa et al., 2009).

There was an abundance of Bacillariophyceae at site $\mathrm{P}_{4}$ during the rainy season, directly related with nutrients (particularly total phosphorus), as this site received organic residue (pig dung) from the plankton cultivation ponds. Inorganic nutrients are regarded as the main source of diatom sustenance; past and present studies have focused on the availability and uptake of organic substrates by diatoms as means of diversifying from conventional trophic sources (Loureiro et al., 2009).

There was a high abundance of Chlorophyceae (except for sites $\mathrm{P}_{3}$ and $\mathrm{P}_{4}$ ) in the phytoplankton community and Rotifera, with the exception of $\mathrm{P}_{1}$ and $\mathrm{P}_{2}$ during the dry season. The sites that supply 
water $\left(\mathrm{P}_{1}\right.$ and $\left.\mathrm{P}_{2}\right)$ for the fish farm during the dry season were represented especially by Cladocera (Bosmina sp.) and Copepoda (Argyrodiaptomus furcatus), which are characteristic of the water impounding reservoir, an oligo-mesotrophic environment. In the rainy season, the increase in water flow in the reservoir led to the appearance of Rotifera that predominated in the fish farm throughout the study period. It was also observed that, starting at site $\mathrm{P}_{3}$ during the dry season and site $\mathrm{P}_{4}$ during the rainy season, Rotifera represented over $70 \%$ of total individuals present in the zooplankton community, emphasizing the effect of the management applied at the fish farm, contributing to a favorable environment for r-strategist organisms. High concentrations of total phosphorus, ammonium and high temperature promote species with a fast reproduction rate and short life span, such as Rotifera (Walz et al., 1995).

The continuous water flow at the fish farm, allied with the rainy season, favored water mixture. Thus, greater diversity and richness of zooplankton species was observed in that period. This increased species diversity, as has been observed with moderate disturbances found in many running water ecosystems (Chellappa et al., 2009). Fish farms normally receive great quantities of allochthonous matter, consisting of feed, fertilizers and food remains, but the rainfall, a characteristic phenomenon during the period of higher fish production (extensive feed supply), has a positive effect on fish farms (SipaúbaTavares et al., 2007).

Higher phytoplankton richness starting at site $\mathrm{P}_{3}$ to $\mathrm{P}_{6}$ was influenced by the management applied within the fish farm to feed the fish and fertilize the fish ponds. Fertilization is so far the most useful technique to make up or provide the essential needed nutrients to enhance the natural productivity through production of aquatic biota, which serve either directly or indirectly as fish food (Begum et al., 2007).

Generally, equitability and diversity values for the zooplankton community were low, with a decreasing trend in the dry season. There was a decreasing diversity $\left(\mathrm{H}^{\prime}\right)$ trend from $\mathrm{P}_{1}$ to $\mathrm{P}_{6}$, with variation between 1.14 and 0.16 during the dry season and between 1.45 and 0.69 during the rainy season. The seasonal variation was considered different among the study sites and as a further indication of temporal heterogeneity. A total of 48 taxa for phytoplankton and 23 for zooplankton in the dry season, and 39 and 36 in the rainy season were considered in the fish farm.

The present study clearly indicated that Chlorophyceae Chlorella sp. overwhelmingly dominated during the dry and rainy seasons, except for site $\mathrm{P}_{3}$ in the dry season, when Microcystis sp. was constant and Pormidium sp. was dominant in the phytoplankton community. In the zooplankton community, Rotifera Keratella americana was common at almost all sites during the rainy and dry seasons.

The article emphasized that continuous water flow and the management employed at the fish farm influenced the plankton community, especially at the sites near the larva culture and plankton culture ponds $\left(\mathrm{P}_{3}\right.$ and $\left.\mathrm{P}_{4}\right)$, where pig dung is added and consequently, high levels of nutrients are observed with dominance of Cyanobacteria and presence of Thermocyclops sp. Furthermore, in the effluent $\left(\mathrm{P}_{6}\right)$ of the fish farm there was an increase in species richness and diversity of the phytoplankton community when compared to water inflow $\left(\mathrm{P}_{1}\right.$ and $\left.\mathrm{P}_{2}\right)$ at the fish farm and an increase in species richness, equitability and diversity of the zooplankton community during the rainy season. The composition and structure of plankton communities reveal changes in water quality, especially with regard to organic matter inputs. The latter causes disturbances in the system and provokes destabilizing situations in the communities.

\section{Acknowledgments}

The authors would like to thank the Furnas Hydrobiology and Hatchery Station (MG, Brazil) for its logistical support of the experiment; CNPq (Brazilian Council for Scientific and Technological Development) for its support and funding to Rachel M. Santeiro.

\section{References}

American Public Health Association - APHA, 1989. Standard methods for the examination of water and wastewater. 16 ed. Washington: AWWA. 268 p.

BASUALTO, S., TAPIA, J., CRUCES, F., BERTRAN, C., SHZATTER, R., PENA-CORTES, F. and HANNSTEIN, E., 2006. The effect of physical and chemical parameters on the structure and composition of the phytoplankton community of lake Budi (IX Region, Chile). Journal of the Chilean Chemical Society, vol. 51, no. 3, p. 993-999.

BEGUM, M., HOSSAIN, MY., WAHAB, MA., AHMED, ZF., ALAM, MJ., SHAH, MR. and JASMINE, S., 2007. Effects of iso-nutrient fertilization on plankton production in earth ponds 
of Bangladesh. Pakistan Journal Biological Sciences, vol.10, no. 8, p. 1221-1228.

CHELLAPPA, NT., CÂMARA, FRA. and ROCHA, O., 2009. Phytoplankton community: indicator of water quality in the Armando Ribeiro Gonçalves reservoir and Pataxó channel, Rio Grande do Norte, Brazil. Brazilian Journal of Biology, vol. 69, no. 2, p. 241-251.

DHAWAN, A. and LAUR, S., 2002. Pig dung as pond manure effect on water quality, pond production and growth carp in poly-culture system. NAGA ICLARM Quaternary, vol. 25, no.1, p. 11-14.

DODSON, SI., NEWMAN, AL., WILL-WOLF, S. ALEXANDER, ML., WOODFORD, MP. and van EGEREN, S., 2009. The relationship between zooplankton community structure and lake characteristics in temperature lakes (Northern Wisconsin, USA). Journal of Plankton Research, vol. 31, no. 9, p. 93-100.

IMAI, H., CHANG, KH., KUSABA, M. and NAKANO, SI., 2009. Temperature-dependent dominance of Microcystis (Cyanophyceae) species: $M$. aeruginosa and M.wesenbergii. Journal of Plankton Research, vol. 31, no. 2, p. 171-178.

LOBO, E. and LEIGTON, G., 1986. Estructuras comunitarias de las fitocienosis planctonicas de los sistemas de desembocaduras de rios y esteros dela zona central de Chilean. Revista Biología Marina, vol. 22, no. 1, p 1-29.

LOUREIRO, S., JAUZEIN, C., GACÉS, E., COLLOS, Y., CAMP, J. and VAQUÉ, D., 2009. The significance of organic nutrients in the nutrition of Pseudo-nitzschia delicatissima (Bacillariophyceae). Journal of Plankton Research, vol. 31, no. 4, p. 399-410.

LUND, JWG., KIPLING, C. and LE CREN, ED., 1958. The inverted microscope methods of estimating algal numbers and statistical basis of estimation by counting. Hydrobiologia, vol. 11, no. 3-4, p. $143-170$.

MACKERETH, FJH., HERON, J. and TALLING, FJ., 1978. Water analyses: some revised methods for limnologists. London: Kendall Titus Wilson \& Sons. 117 p.

MURPHY, J. and RILEY, JP., 1962. A modified single solution method for the determination of phosphate in natural water. Analytica Chimica Acta, vol. 12, no. 2, p. 162-176.

ODUM, EP. and BARRETT, GW., 2007. Fundamentos de ecologia. São Paulo: Thomson Learning. 612 p.

SAMPAIO, EV., ROCHA, O., MATSUMURATUNDISI, T. and TUNDISI, JG., 2002. Composition and abundance of zooplankton in the limnetic zone of seven reservoirs of the Paranapanema river, Brazil. Brazilian Journal of Biology, vol. 6, no. 3, p. 525-545.

SIPAÚBA-TAVARES, LH., GUARIGLIA, CST. and BRAGA, FMS., 2007. Effects of rainfall on water quality in six sequentially disposed with continuous water flow. Brazilian Journal of Biology, vol. 67, no. 4, p. 643-649.

WALZ, W., SARMA, SSS. and BENKER, U., 1995. Egg size in relation to body size in rotifers: an indication of reproductive strategy? Hydrobiologia, vol. 313/314, p. $165-170$.

ZAR, JH., 1996. Biostatistical analysis. 3 ed. New York: Prentice Hall. 622 p.

ZIMBA, PV., MISCHKE, CC. and BRASHEAR, SS., 2003. Pond age-water column trophic relationships in channel catfish Ictalurus punctatus production ponds. Aquaculture, vol. 219, no. 1-4, p. 291-301.

Received: 01 September 2009 Accepted: 30 April 2010 